\title{
Hygroscopicity of 1:2 Choline Chloride:Ethylene Glycol Deep Eutectic Solvent: A Hindrance to its Electroplating Industry Adoption
}

\author{
John Raymund Brusas, and Eden May B. Dela Pena* \\ Sustainable Electrochemical Technologies Laboratory, Department of Mining, Metallurgical and Materials Engineering, \\ College of Engineering, University of the Philippines Diliman, Diliman, Quezon City, Philippines
}

\begin{abstract}
Deep eutectic solvents have been established as feasible metal electroplating solvent alternatives over traditional toxic aqueous plating baths. However, water, either added intentionally or unintentionally, can significantly influence the solvent's physical properties and performance, thereby hindering its industry application. In this study, the hygroscopicity, or the ability to absorb moisture from the environment, of synthesized ethaline (1:2 choline chloride:ethylene glycol) was investigated. The kinematic viscosity, electrical conductivity, electrochemical window, and water content of ethaline were monitored over a 2-week period. Karl Fischer titration tests showed that ethaline exposed to the atmosphere displayed significant hygroscopicity compared to its unexposed counterpart. ${ }^{1} \mathrm{H}$ NMR spectroscopy revealed that water vapor was readily absorbed at the surface due to the hydrophilic groups present in the ethaline molecule. Water uptake resulted in the decrease in viscosity, increase in electrical conductivity and narrowing of the electrochemical window of ethaline. Solution heating at $100^{\circ} \mathrm{C}$ removed the absorbed moisture and allowed the recovery of the solvent's initial properties.
\end{abstract}

Keywords : Deep Eutectic Solvents, Ionic Liquids, Hygroscopicity, Ethaline

Received : 5 October 2020, Accepted : 10 March 2021

\section{Introduction}

Recent advances in room temperature ionic liquids (RTILs) or deep eutectic solvents (DESs) have created the possibility of replacing the traditional aqueous metal plating electrolytes, which are often toxic and raises concerns on its sustainability and environmental risks [1-3]. DES-based baths offer some good engineering advantages for electroplating by having properties such as low vapor pressure, wide electrochemical window, good electrical conductivity, and high solubility for metal salts [4]. More importantly, they have emerged as a "greener" alternative since a majority of DESs are composed of biodegradable and less toxic compounds. There are four types of DES, classified according to chemical composition [5]: (i) Type 1 consists of quaternary ammonium salts (e.g. choline chloride, $\mathrm{C}_{5} \mathrm{H}_{14} \mathrm{ClNO}$ ) with a metal chloride

*E-mail address: ebdelapena@up.edu.ph

DOI: https://doi.org/10.33961/jecst.2020.01522

This is an open-access article distributed under the terms of the Creative Commons Attribution Non-Commercial License (http://creativecommons.org/licenses/by-nc/4.0) Attribution Non-Commercial License (http://creativecommons.org/licenses/by-nc/4.0)
which permits unrestricted non-commercial use, distribution, and reproduction in any which permits unrestricted non-commercial use, distri
medium, provided the original work is properly cited. salt [e.g. zinc chloride, $\mathrm{ZnCl}_{2}$ ); (ii) Type 2 consists of quaternary ammonium salts with a hydrated metal chloride salt (e.g. aluminum chloride hexahydrate, $\left.\mathrm{AlCl}_{3} \mathrm{H}_{12} \mathrm{O}_{6}\right)$ ); (iii) Type 3 has quaternary ammonium salts mixed with a hydrogen-bond donors (HBD) (e.g. carboxylic acid, amide or an alcohol); and (iv) Type 4 that has a hydrated metal chloride salts with a hydrogen bond donor. These mixtures are often used at eutectic concentrations, where the melting point is lowest [5].

A wide range of DESs have already been proven feasible as plating solutions to deposit a wide range of metals [6], including $\mathrm{Cr}$ [7-9], $\mathrm{Ni}$ [10-14], Zn [15], $\mathrm{Sn}$ [16], $\mathrm{Cu}$ [17], $\mathrm{Ag}$ [18], and Pd metal coatings and some Fe-based [19], $\mathrm{Co} / \mathrm{Cr}$ [20], Ni/Cr [21], Ni/Co/ $\mathrm{Sn}$, and $\mathrm{Fe} / \mathrm{Ni} / \mathrm{Cr}[22]$ alloy systems. Its inherently wide electrochemical window allows electrodeposition of elements such as $\mathrm{Al}, \mathrm{Ge}, \mathrm{Si}$, and $\mathrm{Ta}$, which are challenging to do in traditional aqueous plating techniques [23,24].

One common DES is ethaline, a Type 3 DES created by mixing choline chloride $(\mathrm{ChCl})$ and ethylene glycol (EG) at a 1:2 molar ratio. Ethaline has already 
been employed for the successful electrodeposition of different metals including $\mathrm{Ag}, \mathrm{Cr}, \mathrm{Ni}, \mathrm{Zn}, \mathrm{Cr}-\mathrm{P}, \mathrm{Zn}-\mathrm{Sn}$, and $\mathrm{Cu}$ composites, [7,25], and even for nanomaterials [23]. For example, Pereira et al. [15] observed thin $\mathrm{Zn}$ nanoplatelets growing perpendicular in relation to the electrode surface using an ethaline-based $\mathrm{Zn}$ plating bath. Abbott et al. [13] reported nanocrystalline $\mathrm{Ni}$ deposits created from ethaline-based Ni baths. These deposits exhibited lower surface roughness and higher hardness (100 HV harder) than deposits obtained from traditional aqueous-plated $\mathrm{Ni}$ coatings.

The first-generation ionic liquids have been known to be air and water-sensitive - which limits its applicability in electroplating - while DES have been known to be air-stable and water-tolerant [24]. Recently, more studies have investigated how water uptake influences the different properties of DESs.

The presence of hydrophilic groups in the choline chloride molecule is found to be responsible for the high hydrophilic behavior of most choline chloridebased DES [25-29]. These DESs are also considered hygroscopic, substances that have the tendency to absorb moisture from the atmosphere if left exposed. For example, choline chloride-urea DES (aka reline) can absorb atmospheric moisture up to a water concentration of $40 \mathrm{wt} \%$ [30].

The contamination of DESs with water has a significant effect on its properties. In general, water in DESs affects its chemical structure [31], decreases its kinematic viscosity, increases its electrical conductivity, and notably narrows its electrochemical window (EW). Interestingly, some studies have intentionally added water to DES baths to enhance plating mechanics by taking advantage of the improved conductivity and reduced viscosity $[9,32,33]$.

In contrast, the narrowing of the EW due to presence of water can have substantial consequences for electroplating applications. The electrochemical window defines the range of potential at which the solution is stable, and electroplating is most feasible. A high $\mathrm{EW}$ is desirable to allow plating of metals which has high oxidation potentials and require considerable energy for reduction. The typical EW of water is 1.2 V [24]. Du et al. [30] observed a decrease in the electrochemical window (vs. Ag) from $2.54 \mathrm{~V}$ for pure reline to $1.03 \mathrm{~V}$ when the water content was at $12 \mathrm{wt} \%$. However, at $6 \mathrm{wt} \%$ water, there was no observed change in the EW of reline and improvements viscosity, conductivity, and current efficiency were noted. Nickel deposited under this system exhibited a more uniform and compact surface with numerous finer pyramid-like clusters compared to water-free reline [30]. Bobrova et al. reported that an increase in water content in the $\mathrm{CrCl}_{3}-\mathrm{xH}_{2} \mathrm{O}$ and choline chloride DES contributed to the early onset of hydrogen evolution reaction (HER) and led to a drop in the plating current efficiency. The presence of hydrogen gas is undesirable since it can induce hydrogen embrittlement in the substrate and coating [11]. Furthermore, absorbed water could result to decomposition of DES leading to undesirable side reactions [27].

Though the water-tolerance of DES and the ensuing consequence of water content on its chemical properties are well known, there are few studies that details the moisture uptake of DES at typical ambient conditions. From the aspect of electroplating applications, uncontrolled water uptake of the DES bath can possibly alter deposit properties [29]. This uncertainty in deposit quality can easily hinder the commercial adoption of DES as plating bath by the metals industry. Thus, investigation of DES's water uptake from the environment at different storage conditions is worth investigating and will have practical value to electroplaters. Moreover, techniques for the removal of absorbed water also needs consideration, especially if water degrades the relevant DES properties. Cammarata et al. [34] reported that simple heat treatment can indeed remove moisture in ILs. Li et al. [27] reported the use of $\mathrm{N}_{2}$ bubbling and vacuum drying for moisture removal. Nitrogen bubbling was found to remove water effectively from [HOEMIM]Otf ionic liquid as interpreted from the increase of EW to $5.77 \mathrm{~V}$ from $5.38 \mathrm{~V}$ (fresh sample, with initial water content). Vacuum drying at $60^{\circ} \mathrm{C}$ resulted to an EW of 5.50 V[24]. Du et al. [30] employed solution heating at $80^{\circ} \mathrm{C}$ to remove water from reline containing $40 \mathrm{wt} \%$ water, and reached a low of $1.5 \mathrm{wt} \%$ water content after 18 hours. These simple heat treatment strategies to remove absorbed water and return the water-saturated DES to its starting "dry" state is of great importance to widen the application window of DESs.

Based on the existing studies above, this study presents the room temperature moisture absorption behavior of ethaline (1:2 ChCl:EG) over a period of 14 days at two storage conditions. The ethaline's water content, viscosity, conductivity, and electrochemical window were monitored during this period. 
${ }^{1} \mathrm{H}$ NMR spectroscopy was used to assess the incorporation of water molecules into the chemical structure of ethaline. Lastly, solvent heating was employed as an attempt to remove the absorbed water and recover the initial properties of ethaline.

\section{Methodology}

\subsection{Chemicals, Materials and Synthesis}

Choline chloride $(\mathrm{ChCl})$ crystals (Sigma Aldrich, 98\%) were oven-dried at $70^{\circ} \mathrm{C}$ overnight [35] and ethylene glycol (EG) (Ajax Finechem, $>95 \%$ ) was heated at $70^{\circ} \mathrm{C}$ on a magnetic stirrer (EchoTherm HS10-2, USA) for 3 hours to remove initial water content of the reagents prior to synthesis and moisture uptake experiments.

Ethaline was prepared by mixing $\mathrm{ChCl}$ and $\mathrm{EG}$ at $1: 2$ molar ratio and heated to $70^{\circ} \mathrm{C}$ until a clear, homogeneous liquid had formed. The prepared ethaline was allowed to cool to room temperature.

\subsection{Moisture uptake experiments}

Twenty-five (25) $\mathrm{mL}$ aliquots were transferred into centrifuge tubes $\left(7.07 \mathrm{~cm}^{2}\right.$ exposed area) for assessment of moisture uptake. Half of the samples was capped and stored inside a glass container with silica desiccants at $18-20 \%$ relative humidity $(\mathrm{RH})$, as shown in Fig. 1(a). This condition simulates the case in an electroplating plant where the bath is stored in a controlled condition. The other half was left uncapped and exposed to ambient atmosphere $\left(27^{\circ} \mathrm{C}\right)$ at $55-60 \% \mathrm{RH}$, as shown in Fig. 1(b). This condition simulates typical electroplating plant operating conditions where the electroplating bath is left open and exposed to air.

\subsection{Characterization of DES}

For both storage conditions, the water content, viscosity, electrical conductivity, and electrochemical window of ethaline were monitored over a two-week period (14 days).

Water content (wt \%) was determined using a volumetric Karl Fischer titrator (TitroLine 7500KF, SI Analytics, Germany). Sample solution was manually mixed to homogenize before titrating. Three drops or $0.08-0.1 \mathrm{~g}$ of sample were added to the dry methanol solvent (HYDRANAL ${ }^{\mathrm{TM}}$, $\max 0.01 \mathrm{~m} \%$ water) and titrated with the appropriate titrant (HYDRANAL $^{\text {TM }}$, 4.5-5.5 mg/mL water) for moisture determination.

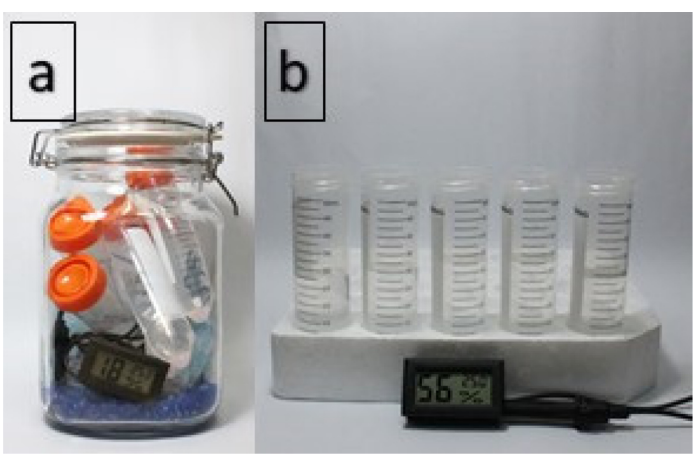

Fig. 1. Setups employed for (a) unexposed and (b) exposed to the atmosphere storage conditions.

Kinematic viscosity was measured at room temperature using a rotational viscometer VISCO $^{\mathrm{TM}}$ 6800, Atago Co., Japan) employing A1S setting (composed of A1 spindle type and S-type beaker) at $150 \mathrm{rpm}$. Electrical conductivity was measured at room temperature using a conductivity meter (LAQUAact-EC120, Horiba, Japan). Measurements were done using a titanium-platinum black conductivity electrode $\left(\mathrm{K}=1 \mathrm{~cm}^{-1}\right)$ (LAQUA 9382-10D, Horiba, Japan) as probe.

Cyclic voltammetry (CV) was performed to observe the changes in the electrochemical window of the DES at different storage conditions. CV measurements were performed at room temperature using a potentiostat (BioLogic SP-150, France) employing a three-electrode system composed of a glassy carbon electrode (area: $7.07 \mathrm{~mm}^{2}$ ) (ALS Co., 002012, Japan) as the working electrode, platinum electrode (area: $2.01 \mathrm{~mm}^{2}$ ) (ALS Co., 002013, Japan) as the counter electrode and silver wire (diameter: $1.5 \mathrm{~mm}$ ) for the reference electrode. The electrodes were polished using 0.5 um alumina before the start of measurement. A scan rate of $10 \mathrm{mV} / \mathrm{s}$ in the scan range of $-1.9 \mathrm{~V}$ to $1.4 \mathrm{~V}$ [27] was employed.

Incorporation of water into the structure of ethaline was observed using proton nuclear magnetic resonance $\left({ }^{1} \mathrm{H}\right.$ NMR) spectroscopy (Agilent $400 \mathrm{MHz}$ NMR, USA) at $400 \mathrm{MHz}$. A 1:2 volume ratio of ethaline sample to deuterated water solvent $\left(\mathrm{D}_{2} \mathrm{O}\right)$ was employed in preparing $600 \mathrm{uL}$ of the sample inside a 5-mm NMR tube (WG-1235-7, Wilmad LabGlass, USA). The ${ }^{1} \mathrm{H}$ NMR spectra were recorded at 32 scans and an average acquisition time of $2 \mathrm{~min}$ utes. 


\subsection{Water removal experiment}

Experiments were conducted to determine if the absorbed water from the environment may be removed from the DES. At the end of the $14^{\text {th }}$ day of storage, the DES was heated at $100^{\circ} \mathrm{C}$ at different durations and allowed to cool to room temperature. The water content was then measured using the Karl Fischer titrator, while the electrochemical window was again assessed via cyclic voltammetry.

\section{Results and Discussion}

\subsection{Moisture absorption of ethaline}

The synthesized ethaline was initially tested for its water content. Results show that the as-prepared solution had $2.4 \mathrm{wt} \%$ water, even after thorough drying of precursor materials. This confirms hygroscopicity or the inherent tendency of ethaline to absorb water from the atmosphere.

Fig. 2 compares the water uptake of ethaline at the different exposure conditions. For the ethaline exposed in air, the water content increased with time. Two regions of water absorption behavior were observed, with the transition point occurring at 112 hrs. Before $112 \mathrm{hrs}$, the linear rate of moisture absorption of ethaline was $11.3 \mathrm{mg} \mathrm{hr}^{-1}$. After which, the rate of absorption slowed by about $20 \%$ to $9.2 \mathrm{mg} \mathrm{hr}^{-1}$. After two weeks, the water content of ethaline in the exposed condition reached a maximum of $14.3 \mathrm{wt} \%$, which is about 6 times higher than its initial value.

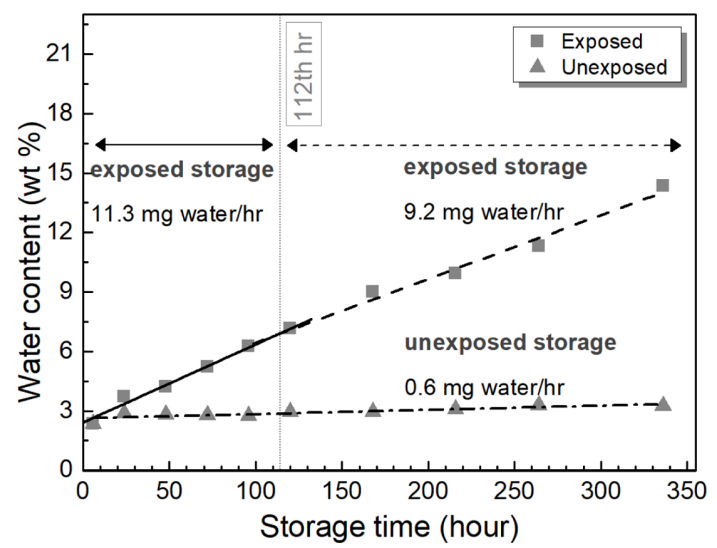

Fig. 2. Water content (wt \%) of ethaline as a function of storage time. Exposure to the atmosphere of ethaline resulted to a high moisture uptake in the span of 14 days.
In contrast, the moisture absorption rate of the unexposed ethaline is significantly lower than its exposed counterpart. The absorption rate was essentially steady at only $0.6 \mathrm{mg} \mathrm{hr}^{-1}$. At the end of two weeks, the moisture content was $3.3 \mathrm{wt} \%$.

${ }^{1} \mathrm{H}$ NMR spectroscopy was employed to determine which functional groups in ChCl-EG complex were responsible for moisture absorption. Measurements were taken at certain time points over a 14-day period. The relevant spectra are shown in Fig. 3, and the corresponding chemical shifts (ppm) and their associated hydrogen type are listed in Table 1 . The peaks of the aliphatic hydrogens in $\mathrm{ChCl}$ and $\mathrm{EG}$ overlapped at 3.35-3.40 ppm [37,38] while the rest are separated and can be identified: 3.05-3.10 (s, 9 $\mathrm{H}$ 's, $-\mathrm{CH}_{3}$ of $\left.\mathrm{ChCl}\right), 3.50-3.55(\mathrm{~s}, 1 \mathrm{H},-\mathrm{OH}$ of $\mathrm{ChCl})$, 3.90-3.95 (m, 2 H's, O-CH ${ }_{2}$ of $\mathrm{ChCl}$ ), 4.60-4.65 (s, 2 H's, water), and 5.555 (d, -OH in EG) [38,39].

Significantly increasing peak intensities observed on chemical shifts (d) 3.05-3.10, and (a) 3.50-3.55 are interpreted to be the functional groups where water is likely to form hydrogen bonds and/or dipoledipole interactions. This result suggest that the presence of hydrophilic groups, $\mathrm{Cl}^{-},-\mathrm{CH}_{3}$, and $-\mathrm{OH}$ $[29,41]$, in the ChCl-EG complex promotes its moisture absorption property, consistent with other works $[26,42,43]$.

The complexation mechanism of $\mathrm{ChCl}$ and $\mathrm{EG}$ is shown in Fig. 4. Hydrogen bonding is established between the chloride anion and the acidic proton on the -OH groups of EG forming a complex anion represented by $[\mathrm{EG}] \mathrm{Cl}^{-}$. Ionic interaction dominates between the choline $\left(\mathrm{Ch}^{+}\right)$, and [EG]Cl- anion forming a ChCl-EG complex [36]. In this molecule, the two $-\mathrm{OH}$ groups and the central $\mathrm{Cl}^{-}$ion are clearly present [26,43]. Chen et al. [26] confirmed that an increase in hydroxyl ions in the molecule enhances a

Table 1. Assigned location and number of hydrogens per chemical shifts observed in ${ }^{1} \mathrm{H}$ NMR.

\begin{tabular}{ccc}
\hline $\begin{array}{c}\text { Chemical shift, } \\
\text { ppm }\end{array}$ & Peak type & Location \\
\hline $3.05-3.10$ & Single & (d) $-\mathrm{CH}_{3}$ of $\mathrm{ChCl}$ \\
$3.35-3.40$ & Multiple & $-\mathrm{CH}_{2}$ - of (c) $\mathrm{ChCl}$ and (f) EG \\
$3.50-3.55$ & Single & (a) $-\mathrm{OH}$ of $\mathrm{ChCl}$ \\
$3.90-3.95$ & Multiple & (b) $\mathrm{O}-\mathrm{CH}_{2}$ - of $\mathrm{ChCl}$ \\
$4.60-4.65$ & Single & (g) water
\end{tabular}



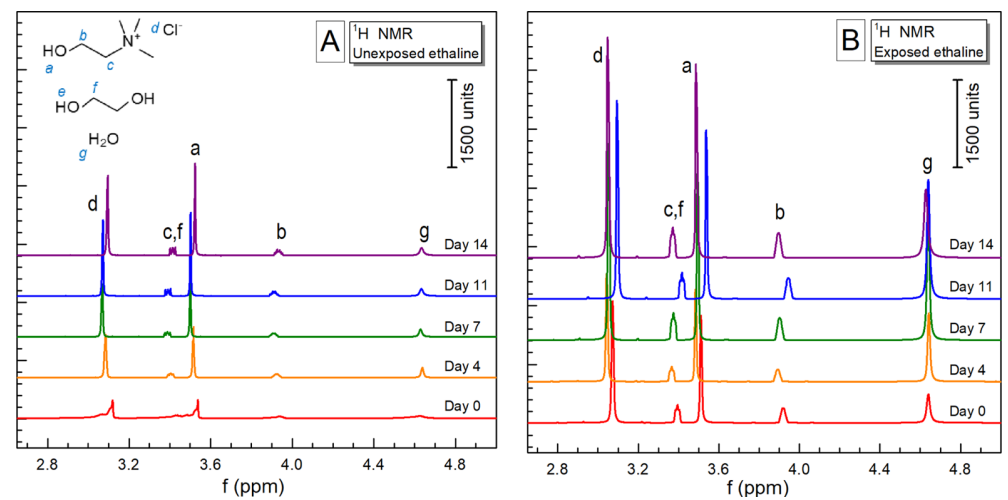

Fig. 3. ${ }^{1} \mathrm{H}$ NMR spectra of (a) unexposed and (b) exposed ethaline for 14 days.

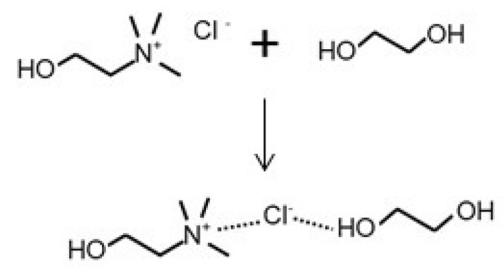

Fig. 4. The chemical reaction involved in the synthesis of choline chloride and ethylene glycol.

DES' water absorption ability. Aside from the number of hydroxyl groups, they observed that other factors such as methyl groups (e.g. ChCl:methylurea vs $\mathrm{ChCl}$ :urea) and chain length can also influence water absorption.

Cao et al. [28] proposed a two-step process mechanism on the moisture absorption of DESs: (1) water vapor molecules from the atmosphere are attracted and absorbed on the surface of the DES, and, after saturation, (2) water moves from the surface to the bulk DES via diffusion as shown in Fig. 5. They observed that a quick shake test reveals the viscosity variation from various levels of the solvent from the surface. This is interpreted as the diffusion of watersaturated layers down to the bulk solution.

Fig. 2 suggests a lowering of the moisture absorption rate upon prolong exposure providing an insight on the water saturation capacity of DESs. Du et al. [30] noted that a hygroscopic $\mathrm{ChCl}$ :urea mixture absorbed a wt $\%$ moisture ( $40 \mathrm{wt} \%)$ that was equivalent to the relative humidity $(\sim 40 \% \mathrm{RH})$ of the test environment. This suggests that it is likely that the rate of moisture uptake of the ethaline will slow down beyond the 14-day period and will absorb a maximum of $18 \mathrm{wt} \%$ water similar to the test room's RH.

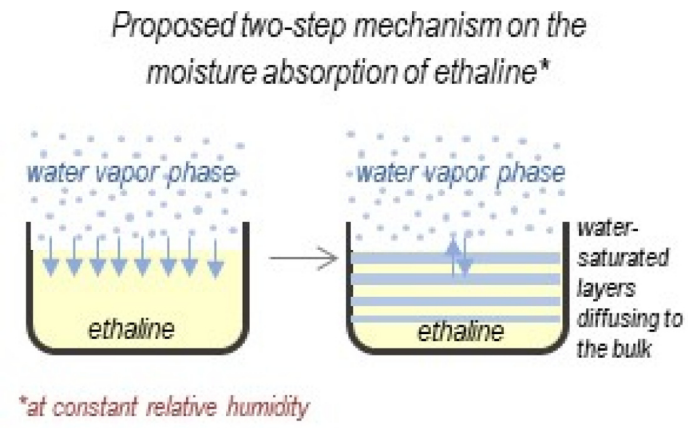

Fig. 5. Water molecules move from the vapor phase and are absorbed on the surface of ethaline then water moves from the surface to the bulk via diffusion (Adopted from [28]).

The negligible change in the water content of the ethaline stored in the controlled environment gives a simple procedure on how to control or limit the hygroscopicity of the DES. This procedure may be considered by future electroplating plants that will deal with hygroscopic ILs.

\subsection{Effect of water on the physical properties of ethaline}

The kinematic viscosity and electrical conductivity of the ethaline at the different storage conditions were also measured within a 14-day period, and results are shown in Fig. 6. The freshly synthesized ethaline was initially assessed and exhibited a viscosity of $39.5 \mathrm{mPa}-\mathrm{s}$ and electrical conductivity of $8.8 \mathrm{mS} \cdot \mathrm{cm}^{-1}$. Published values for the viscosity and conductivity of ethaline are $36-37 \mathrm{mPa} \cdot \mathrm{s}[5,44]$ and $7.61 \mathrm{mS} \cdot \mathrm{cm}^{-1}$ [5], respectively. It is likely that the deviation observed here from other published values 

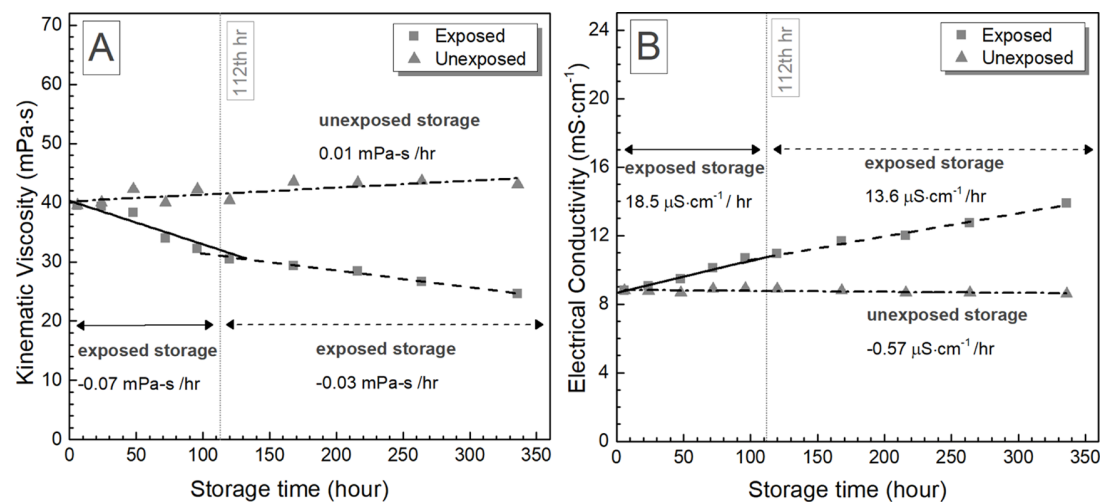

Fig. 6. The influence of water on the (a) kinematic viscosity and (b) electrical conductivity of ethaline for 14-day storage monitoring at unexposed and exposed to atmosphere conditions.

can be attributed to the difference in the water content of ethaline due to difference in fabrication and purification routes. Thus, this observation suggests the importance of including a report on the water content of DESs for every production.

For the ethaline exposed in air (Fig. 6(a)), results show a notable decrease in viscosity, with two linear decline rates defined at the same $112 \mathrm{hrs}$ transition point. The rate of viscosity decline was initially 0.07 $\mathrm{mPa} \cdot \mathrm{s} \mathrm{hr}^{-1}$, and decreased to $0.03 \mathrm{mPa} \cdot \mathrm{s} \mathrm{hr}^{-1}$ after 112 hours. After 14 days, the viscosity of ethaline was lower by about $38 \%$, from $39.5 \mathrm{mPa} \cdot \mathrm{s}$ to $24.6 \mathrm{mPa} \cdot \mathrm{s}$. In contrast, the unexposed ethaline exhibited negligible change in viscosity.

Fig. 6(b) indicate that the electrical conductivity of the ethaline exposed to the atmosphere steadily increased over the 14-day period. Again, two regions of conductivity enhancement were observed. Initially, the conductivity increased at a rate of $18.5 \mu \mathrm{Scm}^{-1} \mathrm{hr}^{-1}$. After 112 hours, the rate slowed to $13.6 \mu \mathrm{Scm}^{-1}$ per hour. At the end of 2 weeks, the electrical conductivity has increased by about $37 \%$ (i.e. from of $13.9 \mathrm{mScm}^{-1}$ to $8.8 \mathrm{mScm}^{-1}$ ). For the unexposed ethaline, there were no significant changes in conductivity observed.

The properties that dictate the mass and charge transport efficiency of an electrolyte are its kinematic viscosity and electrical conductivity. Du et al. [30] was also able to confirm the relationship between electrical conductivity $(s)$ and fluidity $(h)$ in DESs using the equation:

$\sigma \eta=$ constant
Clearly, there is a strong correlation between the observed changes in viscosity and conductivity to the water uptake of ethaline during the test period, as seen in Fig. 2 and Fig. 6. The same bi-modal behaviour was observed, coinciding at a similar transition point (112 hrs).

This relationship between the viscosity and conductivity of the DES and water content may be explained by the hole theory model $[8,41]$. According to this theory, DESs contain void spaces arising from thermally generated fluctuations in local density, which have random size and position and are constantly moving. An ion can only move if it is adjacent to a hole of equal or larger size. Therefore, an ion's mobility, and consequently the DES' viscosity and conductivity, depends on having these appropriately-sized voids or spaces. Bobrova et al. [8] proved that increasing water content of DES resulted to a significant increase in the average void radius, which then promotes the mobility of ions in the system. D'Agostino et al. [45] also reported on the diffusivity of the components of ethaline as influenced by water content. In pure ethaline, the EG molecule moves faster since the relatively large size of the $\mathrm{ChCl}$ molecule reduces its mobility. In water-bearing ethaline, both the diffusivity of $\mathrm{EG}$ and $\mathrm{ChCl}$ is increased, indicating the strong influence of water on the inter-molecular interactions of the ethaline's components.

\subsection{Effect of water on the electrochemical window of ethaline}

The electrochemical window (EW) plays a crucial role in using DESs in the electrodeposition of metals 


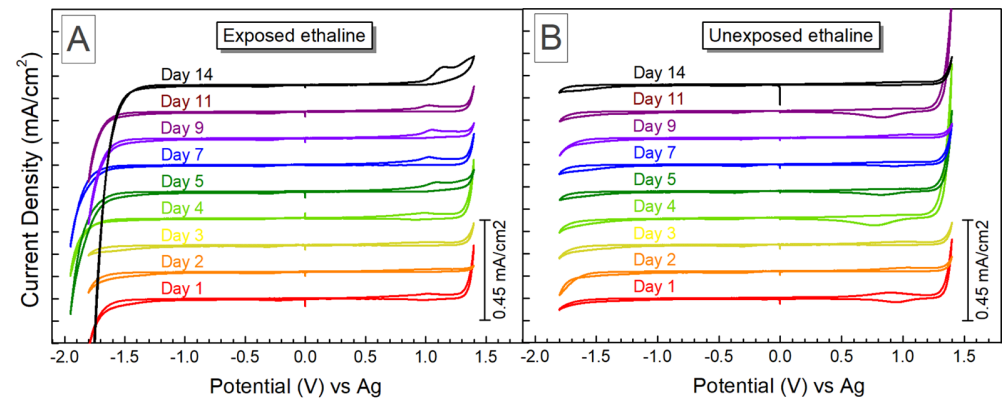

C

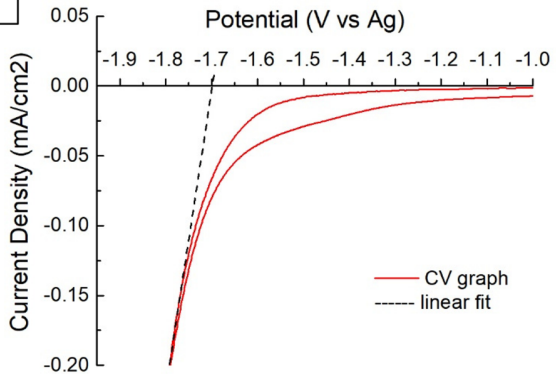

Fig. 7. The evolution of the electrochemical window over the course of 14 days at two different storage conditions, (A) Exposed and (B) Unexposed; (C) Linear fit method was used to determine the electrochemical limits of the electrolyte.

Table 2. Summary of cathodic and anodic limits obtained from CV plots for the exposed and unexposed ethaline storage

\begin{tabular}{ccccccc}
\hline \hline \multirow{2}{*}{ Duration } & \multicolumn{3}{c}{ Exposed } & \multicolumn{3}{c}{ Unexposed } \\
\cline { 2 - 7 } & $\mathrm{E}_{\text {red }}(\mathrm{V})$ & $\mathrm{E}_{\text {ox }}(\mathrm{V})$ & $\mathrm{EW}(\mathrm{V})$ & $\mathrm{E}_{\text {red }}(\mathrm{V})$ & $\mathrm{E}_{\text {ox }}(\mathrm{V})$ & $\mathrm{EW}(\mathrm{V})$ \\
\hline Day 1 & -1.7 & 1.38 & 3.08 & -1.8 & 1.35 & 3.15 \\
Day 2 & -1.75 & 1.4 & 3.15 & -1.7 & 1.4 & 3.1 \\
Day 3 & -1.7 & 1.35 & 3.05 & -1.7 & 1.38 & 3.08 \\
Day 4 & -1.8 & 1.35 & 3.15 & -1.7 & 1.35 & 3.05 \\
Day 5 & -1.75 & 1.35 & 3.1 & -1.7 & 1.35 & 3.05 \\
Day 7 & -1.78 & 1.35 & 3.13 & -1.7 & 1.35 & 3.05 \\
Day 9 & -1.65 & 1.35 & 3 & -1.75 & 1.35 & 3.1 \\
Day 11 & -1.65 & 1.35 & 3 & -1.7 & 1.32 & 3.02 \\
Day 14 & -1.55 & 1.3 & 2.85 & -1.75 & 1.35 & 3.1 \\
\hline
\end{tabular}

and alloys. EW is a good indicator of the bath's electrochemical stability. The EW is defined as the electrochemical potential range over which the electrolyte is neither reduced nor oxidized at an electrode, and is determined from the equation [24]:

$$
E W=\left|E_{\text {red }}-E_{o x}\right|
$$

where $E_{\text {red }}$ is defined as the onset of the reduction potential (cathodic limit), and $E_{\mathrm{ox}}$ is defined as the onset of the oxidation potential (anodic limit). The cathodic and anodic potential limits were obtained via linear fit method recommended elsewhere. The potential limit for either cathodic or anodic reactions is defined as the intersection of the linear fit of the current-voltage relationship after the onset of electrochemical reduction of the electrolyte at the potential axis positioned at $0 \mathrm{~mA} / \mathrm{cm}^{2}$ (Fig. 7c) $[46,47]$. 


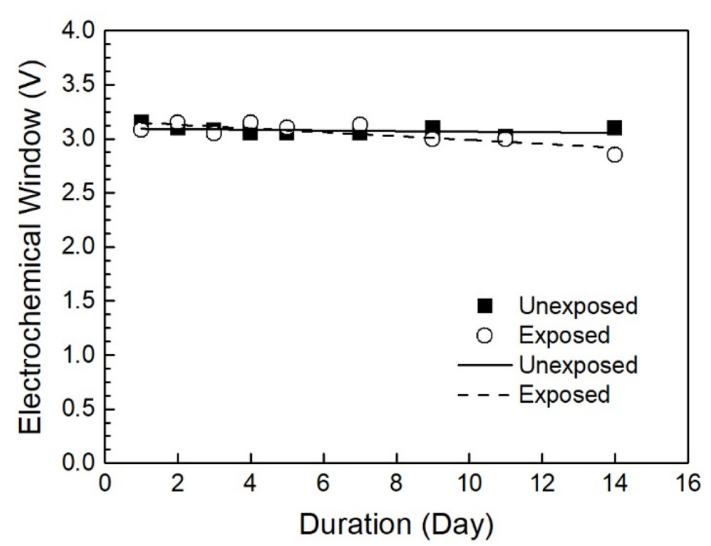

Fig. 8. Evolution of the electrochemical window (EW) of ethaline within 14 days of storage at unexposed and exposed to the atmosphere conditions.

The obtained values for the cathodic and anodic potentials are summarized in Table 2.

Fig. 7 presents the cyclic voltammogram measurements of the ethaline stored at different conditions over the 14-day test period, while Fig. 8 is the corresponding plot of the derived $\mathrm{EW}$ as a function of storage time. The as-prepared ethaline reveals a broad electrochemical window of $3.15 \mathrm{~V}$. The cathodic limit potentials were determined by the reduction of $\mathrm{Ch}^{+}$to triethylamine at about $-1.8 \mathrm{~V}(\mathrm{vs} \mathrm{Ag})$, according to the reaction [48]:

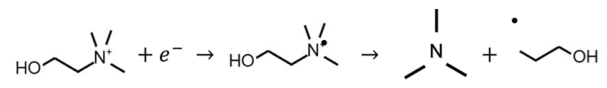

and the anodic limit potential from the oxidation of $\mathrm{Cl}^{-}$to $\mathrm{Cl}_{2}$ gas at about $1.35 \mathrm{~V}$ (vs Ag), [48]:

$$
C l^{-}-e^{-} \rightarrow \frac{1}{2} \mathrm{Cl}_{2}(g)
$$

For the ethaline exposed to the environment, a narrowing of the EW was observed over time. The specimen exhibited a $7.5 \%$ drop in $\mathrm{EW}$, from $3.08 \mathrm{~V}$ to $2.85 \mathrm{~V}$ by the end of Week 2, as seen in Fig. 8. In contrast, the unexposed ethaline had minimal changes in EW, from 3.15 to $3.10 \mathrm{~V}$. Again, this change in EW coincides with the DES' water uptake during the test period. Water can reduce the EW of the DES via the occurrence of electrolytic decomposition reactions, such as the hydrogen evolution reaction $[30,49]$.

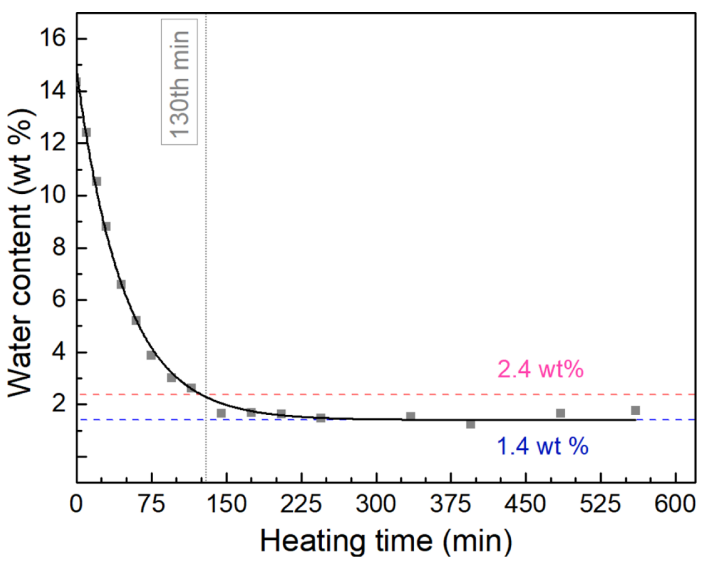

Fig. 9. Water content (wt \%) as a function of solution heating time at $100^{\circ} \mathrm{C}$ for water removal.

\subsection{Water removal via solution heating}

Considering the hygroscopic nature of the DES and the ensuing effects of water absorption on the DES' properties and performance, it is important to adopt simple water removal strategies that could restore the solvent's original properties.

Fig. 9 shows the measured water content in the ethaline that was exposed to air for 14 days after heating at different durations. Evidently, solvent heating at $100^{\circ} \mathrm{C}$ was successful in removing the absorbed water in ethaline. The starting water content value of $2.4 \mathrm{wt} \%$ was obtained after a heating time of about 2 hours, as determined graphically in Fig. 9. Further heating caused the water content in the DES to continue to drop and eventually level. The onset of the water content plateau of $1.4 \mathrm{wt} \%$ started after about 3 hours of heating and was maintained up to 9 hours of heating.

Fig. 10 shows that the EW of the water-laden DES increased after the applied heat treatment from $2.85 \mathrm{~V}$ (EW of Day 14 ethaline sample) to $3.0 \mathrm{~V}$. This value is about $95 \%$ of the EW of the freshly prepared ethaline of $3.15 \mathrm{~V}$. This indicates that heating did not only remove water but also allowed the successful recovery of the DES properties.

However, prolonged heating of up to 9 hours had an unwanted downside as the $\mathrm{ChCl}$ recrystallized upon cooling down to room temperature. The recrystallization of $\mathrm{ChCl}$ is a strong indication that the heated ethaline has strayed from its eutectic concentration (i.e. 1:2 molar ratio of $\mathrm{ChCl}: \mathrm{EG}$ ). Rusdi et al. 


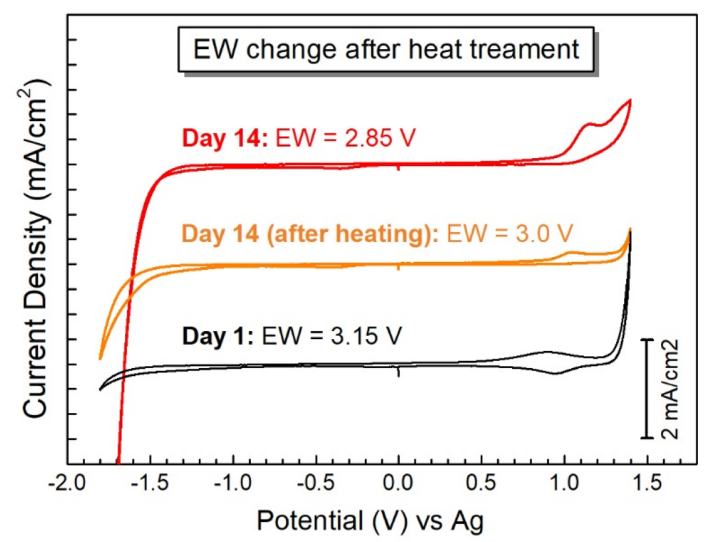

Fig. 10. Heating at $100^{\circ} \mathrm{C}$ was able to nearly restore the electrochemical window of ethaline after it was compromised by the presence of water.

[50] reported that increasing water concentration in an EG-water mixture increases the evaporation rate and decreases the activation energy for the vaporization of the binary system. Hence, it is likely that the combination of water and prolonged heating caused the evaporation of some of the EG and induced the precipitation of $\mathrm{ChCl}$.

The above results confirm the hygroscopicity of ethaline, and the property changes associated with moisture absorption of the DES. As mentioned, the ability of water to positively influence viscosity and conductivity of DES has already been used to improve electroplating results $[9,32,33]$. However, uncontrolled water uptake can lead to unwanted consequences. For example, Protsenko et al. [9] noted that beyond a critical amount of water content, the current efficiency of a ChCl-based chromium plating bath significantly dropped.

Clearly, when used in practical electroplating applications, the handling of ethaline should be an important consideration. Procedures should aim to minimize or control the water intake of the DES to obtain predictable solvent performance. Cao et al. [28] was able to identify other factors affecting hygroscopicity of DES. Temperature promotes diffusion in DES, thereby enhancing moisture absorption. This is important to monitor since the synthesis and electrodeposition steps are done at elevated temperatures for an extended period. Relative humidity, which describes the percentage of water vapor in the air, also has a direct relationship with the DES' water absorption [30]. Thus, humid environments will cause moisture absorption rates to increase. Electroplating operations, especially in the tropical regions, can undergo drastic humidity fluctuations all year round. Therefore, seasonal handling and storage protocols for DESs should be developed. A large, exposed surface area of the DESs will promote hygroscopicity and the faster incorporation of moisture into its bulk system; therefore, capped and narrow-opening mixing, electroplating and storage tanks are suggested for use in this situation.

Water removal strategies must also be integrated in this operation as this will greatly affect the recyclability and cost of the DESs. However, the vaporization mechanism of these DESs must be understood completely as to avoid the evaporation of other components, such as observed in the current study where $\mathrm{ChCl}$ recrystallized due to $\mathrm{EG}$ evaporation. These deviations from the eutectic composition will subsequently cause alterations in the properties and performance of the solvent.

\section{Conclusions}

The hygroscopicity of synthesized ethaline was investigated under two storage conditions, one exposed to the atmosphere and another unexposed or sealed, over a two-week period. When exposed to air, ethaline displayed notable hygroscopicity. Initially the rate of water uptake was high. At about the 4-day mark, the rate of uptake slowed by about $20 \%$. Minimal water uptake was observed in the sealed or unexposed ethaline. ${ }^{1} \mathrm{H}$ NMR spectroscopy revealed that ethaline's considerable hygroscopicity may be traced to the presence of hydrophilic groups, $\mathrm{Cl}^{-}$and $-\mathrm{OH}$, in the ethaline molecule.

With the increase in water content, the ethaline's viscosity decreased while the conductivity increased. This is likely due to water's ability to increase molecule separation distance and expand the hole volume in ethaline, as well as its effects on the ChCl-EG molecular interactions. Furthermore, the EW of the exposed ethaline was reduced as a consequence of water uptake. This decrease in EW was due to the occurrence of water decomposition reactions such as the hydrogen evolution reaction.

Solution heating at $100^{\circ} \mathrm{C}$ was able to remove $80 \%$ of the absorbed water within two hours, and the EW was restored to near original values. 
Hygroscopicity has been proven to affect the properties and possible electroplating performance of ethaline, and this likely applies to most of the DES that are hydrophilic by nature. Hence, appropriate handling, storage, and water removal strategies should be developed to acquire predictable performance and enhance the DES' viability in commercial applications.

\section{Declarations}

\subsection{Funding}

The authors would like to acknowledge the Philippine Council for Industry, Energy, and Emerging Technology Research and Development (PCIEERD) of the Department of Science and Technology (DOST) funded project entitled "Establishing an Environment-Friendly Chromium Plating Process using New Generation Ionic liquids" and the Science Education Institute - DOST.

\section{Conflicts of interest}

The authors declare that there is no conflict of interest.

\section{Acknowledgements}

The authors would like to acknowledge the Philippine Council for Industry, Energy, and Emerging Technology Research and Development (PCIEERD) of the Department of Science and Technology (DOST) funded project entitled "Establishing an Environment- electrodeposition from Ionic Liquids 1Friendly Chromium Plating Process using New Generation Ionic liquids" and the Science Education Institute - DOST.

\section{References}

[1] A. Abbott, F. Endres and D. R. Macfarlane, Electrodeposition from Ionic Liquids, 2017.

[2] A.P. Abbott, and K.J. McKenzie, Phys. Chem. Chem. Phys., 2006, 8(37), 4265-4279.

[3] S. Mishra, and R. N. Bharagava, J. Environ. Sci. Heal. Part $C$, 2016, 34(1), 1-32.

[4] A. P. Abbott, K. S. Ryder, and U. König, Trans. $I M F$, 2008, 86(4), 196-204.

[5] E. L. Smith, A. P. Abbott, and K. S. Ryder, Chem. Rev, 2014, 114(21), 11060-11082.

[6] L. I. N. Tomé, V. Baião, W. da Silva, and C. M. A.
Brett, Appl. Mater. Today, 2018, 10, 30-50.

[7] J. Zhang, C. Gu, Y. Tong, J. Gou, X. Wang, and J. Tu, RSC Adv., 2015, 5(87), 71268-71277.

[8] L. S. Bobrova, F. I. Danilov, and V. S. Protsenko, J. Mol. Liq., 2016, 223, 48-53.

[9] V. S. Protsenko, L. S. Bobrova, A. S. Baskevich, S. A. Korniy, and F. I. Danilov, J. Chem. Technol. Metall., 2018, 53(5), 906-915.

[10] A. P. Abbott, K. El Ttaib, K. S. Ryder, and E. L. Smith, Trans. IMF, 2008, 86(4), 234-240.

[11] E. A. Mernissi Cherigui, K. Sentosun, P. Bouckenooge, H. Vanrompay, S. Bals, H. Terryn, and J. Ustarroz, $J$. Phys. Chem. C, 2017, 121(17), 9337-9347.

[12] A. A. Kityk, D. A. Shaiderov, E. A. Vasil'eva, V. S. Protsenko, and F. I. Danilov, Electrochim. Acta, 2017, 245, 133-145.

[13] A. P. Abbott, A. Ballantyne, R. C. Harris, J. A. Juma, and K. S. Ryder, Electrochim. Acta, 2015, 176, 718-726.

[14] A. P. Abbott, A. Ballantyne, R. C. Harris, J. A. Juma, and K. S. Ryder, Phys. Chem. Chem. Phys., 2017, 19(4), 3219-3231.

[15] N. M. Pereira, C. M. Pereira, J. P. Araújo, and A. Fernando Silva, J. Electroanal. Chem., 2017, 801, 545551.

[16] S. Salomé, N. M. Pereira, E. S. Ferreira, C. M. Pereira, and A. F. Silva, J. Electroanal. Chem., 2013, 703, 80-87.

[17] P. Sebastián, E. Vallés, and E. Gómez, Electrochim. Acta, 2014, 123, 285-295.

[18] P. Sebastián, E. Vallés, and E. Gómez, Electrochim. Acta, 2013, 112, 149-158.

[19] F. Zhao, S. Franz, A. Vicenzo, M. Bestetti, F. Venturini, and P. L. Cavallotti, Electrochim. Acta, 2013, 114, 878888.

[20] G. Vargas, J. Lopez, A. P. García, and J. Cerda, ISES Conf. Proc., 2016.

[21] A. Abdullah and A. Barzinjy, ZANCO J. Pure Appl. Sci., 2016, 28(2), 47-55.

[22] G. Saravanan and S. Mohan, Int. J. Electrochem. Sci., 2011, 6, 1468-1478.

[23] E.L. Smith, J.C. Barron, A.P. Abbott, and K.S. Ryder, Anal. Chem., 2009, 81(20), 8466-8471.

[24] F. Endres, S. Zein, and E. Abedin, Phys. Chem. Chem. Phys., 2006, 8(18), 2101-2116.

[25] F. Liu, Y. Deng, X. Han, W. Hu, and C. Zhong, J. Alloys Compd., 2016, 654, 163-170.

[26] Y. Chen, D. Yu, W. Chen, L. Fu, and T. Mu, Phys. Chem. Chem. Phys., 2019, 21(5), 2601-2610.

[27] Q. Li, J. Jiang, G. Li, W. Zhao, X. Zhao, and T. Mu, Sci. China Chem., 2016, 59(5), 571-577.

[28] Y. Cao, Y. Chen, X. Sum, Z. Zhang, and T. Mu, Phys. Chem. Chem. Phys., 2012, 14(35), 12252-12262.

[29] Y. Koga, P. Westh, K. Nishikawa, and S. Subramanian, J. Phys. Chem. B, 2011, 115(12), 2995-3002.

[30] C. Du, B. Zhao, X. Chen, N. Birbilis, and H. Yang, Sci. Rep., 2016, 6(1), 1-14

[31] O. S. Hammond, D. T. Bowron, and K. J. Edler, Angew. 
Chem. Int. Ed., 2017, 56(33), 9782-9785.

[32] V.S. Protsenko, L.S. Bobrova, A.S. Baskevich, S.A. Korniy, F.I. Danilov, J. Chem. Technol. Metall., 2018, 53(5), 906-915.

[33] D.C. McCalman, L. Sun, Y. Zhang, J.F. Brennecke, E.J. Maginn, W.F. Schneider, J. Phys. Chem. B, 2015, 119(19), 6018-6023.

[34] L. Cammarata, S. G. Kazarian, P. A. Salter, and T. Welton, Phys. Chem. Chem. Phys., 2001, 3(23), 51925200 .

[35] A.M.M. Sousa, H.K.S. Souza, L. Nicholas, C-K Liu, M.P. Goncalves, and LS Liu, Carbohydr. Polym., 2014, 111, 206-214.

[36] A. P. Abbott, G. Capper, D. L. Davies, R. K. Rasheed, and V. Tambyrajah, Chem. Commun., 2003, (1), 70-71.

[37] I. Delso, C. Lafuente, J. Muñoz-Embid, and M. Artal, J. Mol. Liq., 2019, 290, 111236.

[38] C. D. Agostino, R. C. Harris, A. P. Abbott, F. Gladden, and M. D. Mantle, Phys. Chem. Chem. Phys., 2011, 13(48), 21383-21391.

[39] N. R. Babij, E. O. Mccusker, G. T. Whiteker, B. Canturk, N. Choy, L. C. Creemer, C. V. De Amicis, N. M. Hewlett, P. L. Johnson, J. A. Knobelsdorf, F. Li, B. A. Lorsbach, B. M. Nugent, S. J. Ryan, M. R. Smith, Q. Yang, Org. Process Res. Dev., 2016, 20(3), 661-667.
[40] R. J. Abraham, J. J. Byrne, L. Griffiths, and R. Koniotou, Magn. Reson. Chem., 2005, 43, 611-624.

[41] A. P. Abbott, J. C. Barron, K. S. Ryder, and D. Wilson, Chem. A Eur. J., 2007, 13, 6495-6501.

[42] O. S. Hammond, D. T. Bowron, and K. J. Edler, Angew. Chem. Int. Ed., 2017, 129, 9914-9917.

[43] A. Pandey, R. Rai, M. Pal, and S. Pandey, Phys. Chem. Chem. Phys., 2014, 16, 1559-1568.

[44] G. Garc, S. Aparicio, R. Ullah, and M. Atilhan, Energy Fuels, 2015, 29, 2616-2644.

[45] C. D'Agostino, L. F. Gladden, M. D. Mantle, A. P. Abbott, E. I. Ahmed, A. Y. M. Al-Murshedi, and R. C. Harris, Phys. Chem. Chem. Phys., 2015, 17, 2138321391.

[46] M. Mousavi, A. Dittmer, and B. Wilson, J. Electrochem. Soc., 2015,162(12), A2250-A2258.

[47] E. Olson, and P. Buhlmann, J. Electrochem. Soc., 2013, 160(2), A320-A323.

[48] D. Yue, Y. Jia, Y. Yao, J. Sun, and Y. Jing, Electrochim. Acta, 2012, 65(30), 30-36.

[49] H. Ohno, Electrochemical Aspects of Ionic Liquids, 2005.

[50] M. Rusdi, Y. Moroi, and H. Nakahara, Langmuir, 2005, 21(16), 7308-7310. 